\title{
Construction and Functional Analysis of beclin1 promoter luciferase reporter plasmid
}

\author{
Doudou $\mathrm{Li}^{1, \mathrm{a}}$, Na Huang ${ }^{1, \mathrm{~b}}$, Xiaomei $\mathrm{Li}^{1, \mathrm{c}}$, Hao Zhou ${ }^{1, \mathrm{~d}}$ and Nan Wang ${ }^{1, \mathrm{e}}$ \\ ${ }^{1}$ Key Laboratory of Industrial Microbiology, Ministry of Education and Tianjin City, College of \\ Biotechnology, Tianjin University of Science and Technology \\ alidou0313@163.com, b541810464@qq.com, 868898501@qq.com, \\ dzhouhao@tust.edu.cn, ${ }^{e}$ wn929@tust.edu.cn
}

\begin{abstract}
Keywords: autophagy, beclin1, E2F1, promoter activity, luciferase assay
Abstract. Autophagy is a conserved process in eukaryotic cells, which is induced by extracellular and intracellular signals, degrading biological macromolecules and damaged organelles through fusion with lysosomes. Beclin1, which is homologous to the yeast autophagy related gene Atg6, acts as a key gene for autophagy. It combines with the class III phosphatidylinositol 3-kinase (PtdIns3K) and Vps34 to form the key complex in the induction of autophagy. E2Fs transcription factors are known to be involved in the regulation of cell cycle progression. By analyzing the promoter sequence of beclin1, we found that there were two E2F1 binding sites within the promoter. In the present study, the gene fragment of human beclin1 gene promoter containing E2F1 was cloned into empty vector pGL3-Basic to construct beclin1 promoter luciferase reporter plasmid. E2F1 expression plasmids, together with beclin1 promoter-luc plasmids were transfected into COS-7 cells, and then luciferase assay was performed to analyze the effects of E2F1 on regulating the promoter activity of beclin1. The results showed that E2F1 can activate the transcription of beclin1. Constuction of beclin1 promoter luciferase reporter plasmid will provide the theory basis for investigating the function of beclin1 in autophagy.
\end{abstract}

\section{Introduction}

Autophagy is a self-degradation pathway, which occurs under some conditions, including oxidative stress, ceramide, and endoplasmic reticulum stress [1]. In the process, some damaged proteins or organelles are packaged by the autophagic vesicles of the bilayer membrane structure, and then the materials are transported into the lysosomes to degrade and recycle [2]. Beclin1 is one component of the PI (3) kinase III (PI (3) KC3) lipid kinase complex, and is the first human protein shown to be indispensable for autophagy [3]. The autophagic core complex mainly includes beclin1, UV radiation resistance-associated gene protein (UVRAG), the lipid kinase Vps34. To identify upstream transcription factors of beclin1 will contribute to find the new regulator of autophagy and understand the role of beclin1 in autophagy.

E2F transcription factor can regulate the expression of genes, such as proto-oncogenes and cell cycle progression [4]. As a transcription factor, E2F1 can bind to TTTC[CG]CGC site and activate the transcription of target gene [5]. E2F1 was reported to activate the transcription and expression of three autophagy genes including autophagy-related gene-1 (ATG1), microtubule-associated protein-1 light chain-3 (LC3) and damage-regulated autophagy modulator (DRAM) [6]. By analyzing the promoter sequence of beclin1, we have found that there are two E2F1 binding sites within the promoter of beclin1. Thus, in this paper, luciferase reporter gene plasmids containing beclin1 promoter was constructed and the role of E2F1 in regulating the transcription of beclin1 was investigated by luciferase assay.

\section{Materials and Methods}

Cell Culture and Genomic extraction. COS-7 cells (ATCC) and Hela cells (ATCC) were cultured in Dulbecco's Modified Eagle Medium/Nutrient Mixture F-12 (DMEM/F-12, GIBCO) containing 
$10 \%$ FBS (Atlanta Biologicals), penicillin $(100 \mathrm{U} / \mathrm{mL})$ and streptomycin $(100 \mathrm{U} / \mathrm{mL})$ at $37^{\circ} \mathrm{C}$ in a $5 \%$ $\mathrm{CO}_{2}$ incubator. The genomic DNA of Hela cells was extracted in Universal Genomic DNA Kit (CWBIO) according to the instructions.

Plasmid Construction. The beclin1 promoter containing E2F1 binding site (from -124 to -117 and -23 to -16) was amplified from genomic DNA of Hela cells by PCR and cloned into a pGL3 luciferase reporter vector. The primers used in PCR reactions were as follows: forward primer CGACGCGTAGCCTCTGCTAATCC, reverse primer - GGAAGATCTCCAGGGTCAGGGAAGG. Amplification conditions for PCR are as follows: predegeneration for $5 \mathrm{~min}$ at $94^{\circ} \mathrm{C}$, denaturation for $30 \mathrm{~s}$ at $94^{\circ} \mathrm{C}$, annealing for $30 \mathrm{~s}$ at $56^{\circ} \mathrm{C}$ and extension for $1 \mathrm{~min} 40 \mathrm{~s}$ at $72^{\circ} \mathrm{C}$. PCR reaction was carried out for 30 cycles.

The PCR products and pGL3-Basic vector were digested with restriction enzyme Mlu I (Thermo) and $\mathrm{Bgl} \mathrm{II}$ (Thermo) at $37^{\circ} \mathrm{C}$ for $1.5 \mathrm{~h}$ and then the gene fragment was linked to pGL3-Basic vector using T4 DNA ligase (Thermo) at $16^{\circ} \mathrm{C}$ for $12 \mathrm{~h}$. The recombinant plasmids were transformed into E.coli DH5 $\alpha$ and confirmed by restriction enzyme digestion and DNA sequencing.

Plasmid transfection. For transfection experiments, COS-7 cells were plated in 24-well dishes when they were $60-70 \%$ confluent. Transfection was carried out using transfection reagent Turbofect (Thermo) following the manual of the manufacturer.

Luciferase Reporter Assays. Luciferase activity assay was performed using the Luciferase Assay System (Promega) according to the instructions. Briefly, 24 hours after transfection, the transfected cells were lysed in Cell Culture Lysis Reagent. $20 \mu \mathrm{L}$ of cell lysate was added into a 96-well enzyme label plate and reading was initiated by the injection of $100 \mu \mathrm{L}$ of Luciferase Assay Reagent into the plate on a Synergy ${ }^{\mathrm{TM}} 4$ (Bioteck). Each sample was examined in duplicate and it was repeated in 3 different experiments.

Statistical analyses. Data were presented as means \pm SD from at least three separate experiments. The significant difference was examined using the Student's test. The minimal level of significance was $\mathrm{P}<0.05$.

\section{Results}

Construction of beclin1 Luciferase Reporter Plasmid. The schematic structure of human beclin1 promoter containing E2F1 binding site was shown in Fig. 1. The beclin1 promoter sequence from -1000 to +200 was cloned from the genomic DNA of the hela cell line.

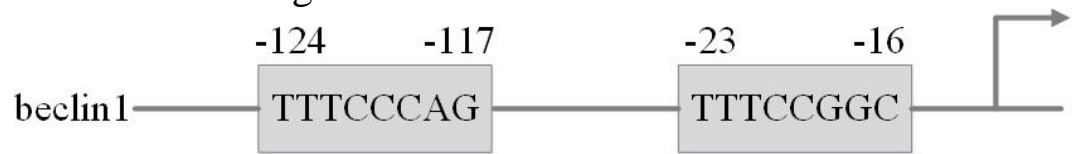

Figure1. A structure schematic of luciferase reporter plasmids containing beclin1 promoter.

To estimate the PCR amplification of beclin1 promoter, agarose gel electrophoresis was performed. As shown in Fig. 2, one band emerged at the site of $1200 \mathrm{bp}$, which represent PCR product of beclin1 promoter.

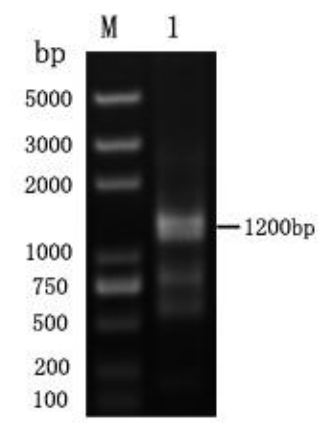

Figure 2. Agarose gel electrophoretic analysis of PCR amplification products (M: DL2000 Plus Marker; 1: PCR products). 
As shown in Fig. 3A, the recombinant plasmids were digested with restriction enzyme Mlu I and $B g l \mathrm{II}$ at $37^{\circ} \mathrm{C}$ for $1 \mathrm{~h}$. Purified vector fragments and PCR fragments were used in a DNA ligation reaction to generate recombinant plasmids.

As shown in Fig. 3B, the recombinant plasmid was digested with $M l u$ I and $B g l \mathrm{II}$ at $37^{\circ} \mathrm{C}$ for $1 \mathrm{~h}$ and $1 \%$ agarose gel electrophoretic was used to analyze the DNA products, the sepharose electrophoresis of DNA showed two major bands: a 1.2-kb band could represent beclin1 gene promoter; a lower than 5.0-kb band could represent a pGL3-Basic vector. Beclin1 promoter luciferase reporter gene plasmid (p-beclin1-Luc) was successfully constructed, confirmed by sequencing.
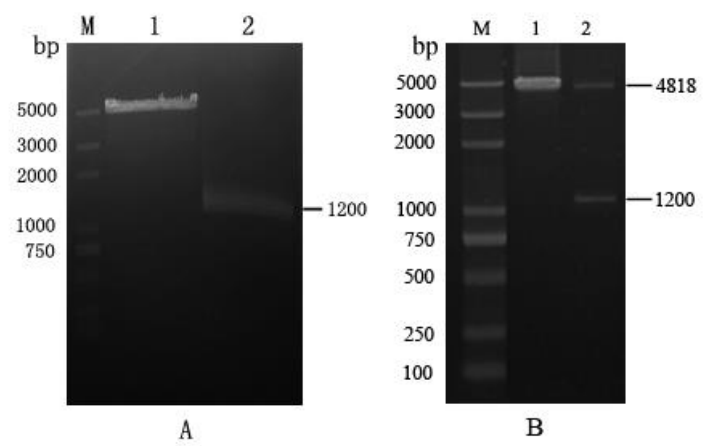

Figure 3. A: Restriction enzyme digestion of pGL3-basic vector and PCR product (M: DL2000 Plus Marker; 1: Double digestion of pGL3-basic vector; 2: Double digestion of beclin1 promoter).

B: Agarose gel electrophoretic analysis of recombinant plasmids. M: DL2000 Plus Marker; 1: pGL3-basic vector; 2: Double digestion of beclin1 recombinant plasmid.

Luciferase Assay. To analyze whether the transcription activity of beclin1 promoter is regulated by E2F1, the beclin1 promoter plasmids were transfected into COS7 cells together with pcDNA3.1 empty vector or pcDNA3.1-E2F1 expressive plasmids for $24 \mathrm{~h}$. As shown in Fig. 4, compared to the control group, the overexpression of E2F1 significantly upregulated the activity of beclin 1 promoter approximately 2-fold, suggesting that E2F1 could activate the transcription of beclin 1 and beclin 1 might be one target gene of E2F1.

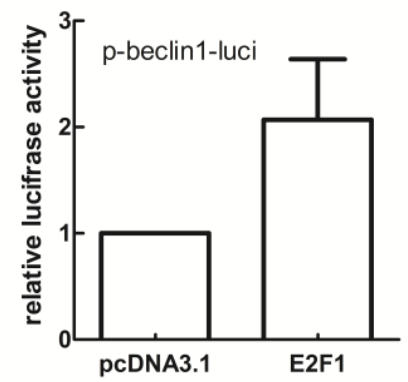

Figure 4. E2F1 increased the transcriptional activity of beclin1 promoter.

\section{Discussion}

Autophagy plays an important role in normal cell growth. It contributes to maintain the balance of intracellular environment stability by removing intracellular excess organelles and proteins. Autophagy is essential to prevent diseases such as tumors, cardiomyopathy, pathogenic microbial invasion. To explore the function of beclin1, as an indispensable gene for autophagy, will provide more knowledge about the role and regulation of autophagy in physiological and pathology.

In this study, beclin1 promoter luciferase reporter plasmid was successfully constructed. Luciferase assay confirmed that E2F1 can transactivate the beclin1 promoter. Other transcription factors such as NFKB [7] and HIF-1 [8] have been shown to up-regulate the expression of beclin1 gene. Similar, Bing Wang et al. also demonstrated that E2F1 can regulate the transcription of beclin1 [9]. Taken with other results, our results preliminarily suggested that E2F1 might play an important role in the regulation of autophagy and related gene expression. And further study is needed to 
illuminate the role of beclin1 in autophagy.

\section{Acknowledgements}

This work was financially supported by the National Natural Science Foundation of China (31171303, 31301073 and 31470816).

\section{References}

[1] Mathiassen SG, De Zio D, Cecconi F: Frontiers in oncology.7(2017), p. 51

[2] Aita VM, Liang XH, Murty VV, Pincus DL, Yu W, Cayanis E, et al.: Genomics.59(1999), p. 59-65

[3] Beth Levine SS, Guido Kroemer: Autophagy.4(2008), p. 600-6

[4] Yang XH, Sladek TL: Gene expression.4(1995), p. 195-204

[5] Zaragoza K, Begay V, Schuetz A, Heinemann U, Leutz A: Molecular and cellular biology.30(2010), p. 2293-304

[6] Polager S, Ofir M, Ginsberg D: Oncogene.27(2008), p. 4860-4

[7] Nivon M, Richet E, Codogno P, Arrigo AP, Kretz-Remy C: Autophagy.5(2009), p. 766-83

[8] Bohensky J, Shapiro IM, Leshinsky S, Terkhorn SP, Adams CS, Srinivas V: Autophagy.3(2007), p. $207-14$

[9] Wang B, Ling S, Lin WC: PloS one.5(2010), p. e10409 